\title{
Nitrogen Isotope Separation by lon Exchange Chromatography
}

\author{
Xingcheng Ding and Xunyue Liu \\ Additional information is available at the end of the chapter
}

http://dx.doi.org/10.5772/51311

\section{Introduction}

Enriched stable isotopes of many elements have been widely used in many aspects and such isotopes have been primarily used as the tracer for agricultural and biochemical studies and the availability of the isotopically labeled compounds have been now extensively increasing. Chemical exchange separation of isotopes is based on the equilibrium fractionation of isotopes between two phases, ion exchange isotope separation which is one of the chemical exchange methods, is based on the chemical equilibrium between a stationary phase and a mobile fluid phase. Displacement always results in a sharp boundary between the bands of eluted solute and displacing solute in this method. Displacement chromatographic ion exchange technology for isotope separation is described in this chapter. This chapter mainly divided into two sections. Ion exchange system, ion exchange separation and analysis are introduced in the former section as the basic theory and concept for ion exchange separation. The second section is about the experiment parts of nitrogen isotope separation which include nitrogen isotope application, nitrogen isotope separation process, nitrogen isotopic analysis with mass spectrometry and the obtained results and discussion which were performed by our recent experiments.

\section{Ion exchange system}

Enriched stable isotopes of many elements have been widely used in agriculture, medicine, biochemistry and industry, especially in nuclear industry. Such isotopes have been primarily used as the tracer for agricultural and biochemical studies and the availability of the isotopically labeled compounds have been now extensively increasing. In order to meet the demand, the need of developing a practical cost-effective process has been enlarged. The most heavy stable isotopes are produced in relatively small quantities, many light isotopes 
are used in large quantities. Distillation is used in industry to separation the isotopes of hydrogen, carbon, boron, nitrogen and oxygen. Chemical exchange is the satisfactory technique for separating isotopes of light elements because of their relatively high simple process factors (separation factors).

Chemical exchange separation of isotopes is based on the equilibrium fractionation of isotopes between two phases, i.e. one of the isotopes is concentrated more abundantly in one of the phases than in the other phase if two chemical species are distributed in each phase. In this method, the enrichment of the isotopes concerned is achieved when the simple process of isotope separation is multiplied, by arranging the countercurrent contacting of two substances. The countercurrent contacting of the two different chemical substances is most easily realized in vapor-liquid or liquid-liquid system. The contacting takes place in conventional systems such as packed columns, mixer-settlers, etc. In this process it is necessary to provide reflux at the product end of a chemical exchange plant; the end which the desired isotope being enriched. In general, chemical refluxing is used to complete the conversion of one chemical species into the other at the end of the multi-stage contactor system.

Ion exchange isotope separation, which is one of the chemical exchange methods, is based on the chemical equilibrium between a stationary phase and a mobile fluid phase. In the past, many researchers have studied isotope fractionation on the isotopes both for light and heavy elements in this liquid solid system[1-5] and several isotopes especially for nitrogen isotope have been successfully enriched in laboratory scale by using displacement chromatographic technique [6, 7]. In this process a band of adsorbate is eluted through the column by a displacing solution, which the rate of movement of the band is determined by the equilibrium between solution and adsorbent, not of the material of the band but of the displacing adsorbate. Displacement always results in a sharp boundary between the bands of eluted solution and displacing solution. Thus, the adsorption band moves with keeping a constant band length. During the migration the isotopes in the band are rearranged in order of the distribution coefficients.

\section{Ion exchange separation and analysis}

\subsection{Ion exchange column}

\subsubsection{Batch and column operation}

Ion exchanges are generally employed in the two different modes of operations: batch and column operation. The batchwise operation consists of contacting the whole of an electrolyte solution to be treated with a mass of exchanger and then separating the two phases by means of filtration, decantation, etc. It is quite obvious that for those exchange reactions that do not approach completion, a batchwise operation must be repeated many times before complete transformation is realized. The more unfavorable the equilibrium, the larger the number of stages are necessary for a given degree of exchange or separation. 
The operation of an ion-exchange reaction by leaching the exchanger in a columnwise manner achieves most efficiency the same goal that would take many stepwise stages in a batch method. The column operation is essentially an elaborate multiple batchwise technique in which the uppermost portion of the column is constantly contacting fresh electrolyte whereas the lower portions contact the electrolyte not adsorbed by the upper exchanger. This procedure permits the exchanger bed to become fully exhausted at the top and then gradually downward. Ion exchange chromatography is a technique in which resolution of a mixture is achieved by virtue of differences in migration rates of the components in a column packed with an ion exchanger.

\subsubsection{Chromatographic column operation}

Chromatography was first applied to colored substances where "bands" of different colors can be seen while they move down the column. The chromatographic column operations are generally conducted in the manners of elution development, displacement development, and frontal analysis [8-10]. In the development techniques, a certain amount of the mixture is introduced at the top of the column and is then "developed" or "eluted" by a suitable agent. In frontal analysis, the mixture is continuously fed to the column. The front boundaries of the various components emerge at different times due to the differences in the migration rates.

\subsubsection{Preparation of ion exchange column}

In the analytical applications of ion exchange, it is necessary to utilize uniform particle size ion exchangers in order to avoid irregular flow due to the distribution of particle size. For this purpose, the wet resins in the form of $\mathrm{H}^{+}$were screened prior to the packing. The stainless steel screens of 42, 80, 200 and 400 meshes are used commonly. Then the ion exchanger with water in a beaker was stirred, and poured the slurry of resin into the column.

Under no circumstances must bubble or air be allowed to form in the bed of the ion exchanger beads. In operation, therefore, the bed must never be allowed to drain; the liquid level must not fall below the top of the bed. The water level was kept at $1-2 \mathrm{~cm}$ above the top of the bed during the operation.

The fresh ion exchanger provided by the supplier usually contains impurities, such as low molecular weight organic substances, and metallic ions, such as ferric ions. The new ion exchanger bed was, thus, washed in order to remove these impurities and metallic ions by following manner:

1. The bed is first washed with a ten fold column volume of $2.0 \mathrm{M}$ hydrochloric acid.

2. The same amount of $10 \mathrm{M}$ sodium chloride is then fed into the column.

3. Repeat the process (1) and (2) for three times.

4. Repeat the process (1).

5. The bed is finally rinsed with distilled water until no leakage of $\mathrm{H}^{+}$is recognized. 


\subsubsection{Apparatus}

In the ion exchange chromatography, high pressure resistant pyrex glass columns with a water jacket were used in the studies of isotope separation. The column of which the inside diameters of 8,10 and $20 \mathrm{~mm}$ were prepared so that the operations are able to be conducted up to the column pressure of 50,30 and $20 \mathrm{~kg} / \mathrm{cm}^{2}$ in each column. To feed solutions, the microprocessor controlled, double plunger type titanium high pressurized pumps were used. The Teflon tubing of $1.0 \mathrm{~mm}$ i.d. was employed to connect column and pump. To monitor the column pressure, a pressure gauge with a safety device was placed between the column and the pump. In addition, an air damper was used in order to prevent the piston flow, especially at high flow rate runs, when loading solutions into columns. A schematic diagram of the apparatus used for the ion exchange separation is shown in Figure1. Water jacket was employed to remove the heat of reaction and maintain the column temperature throughout the experiments by circulation the thermostated water.

\section{Nitrogen isotope application}

Enriched isotope of ${ }^{15} \mathrm{~N}$ has been a very valuable commercial product for which there is presently a growing demand in various scientific research and industry applications. In the agricultural field, ${ }^{15} \mathrm{~N}$ has been extensively used to study nitrogen cycling in soil-plant relationships. The fertilizer efficiency, distribution, assimilation and metabolic plants in the plant, symbiotic nitrogen fixation and the behavior of fertilizer in soils have been studied with use of ${ }^{15} \mathrm{~N}$. Adriana et al. evaluated the nitrogen fixing capacity of a range of commercial cultivars of maize (Zea mays L.) by the ${ }^{15} \mathrm{~N}$ isotope-dilution method. Biological nitrogen fixation expressed as percent nitrogen derived from air ranged from 12 to 33 regardless of nitrogen fertilization [11]. Their results demonstrated that maize cultivars obtain significant nitrogen from biological nitrogen fixation, varying by maize cultivar and nitrogen fertilization level. In order to improve yields of crop production in many areas, one strategy is to choose crops with high nitrogen use efficiency that can produce economic yields under limited water supply. Sarr et al. performed the pearl millet (Pennisetum glaucum L.R.Br.) and cowpea (Vigna unguiculata L. Walp.) in sole crops and intercrops systems for the nitrogen use efficiency of applied fertilizers [12]. ${ }^{15} \mathrm{~N}$-labeled urea at rates of $20 \mathrm{~kg} \mathrm{ha}^{-1}$ (sole and intercrop cowpea) and $41 \mathrm{~kg} \mathrm{ha}^{-1}$ (sole millet and intercrop millet) was derived from the nitrogen fertilizer and $84.70 \%$ from nitrogen mineralized in soil. In addition, many other publications reported the ${ }^{15} \mathrm{~N}$ application in agroecological system [13-17]. In the industrial application, growing attention has been recently placed on the use of ${ }^{15} \mathrm{~N}$ isotope for the nitride fuels of FBRs (Fast Breeders Reactors) because of their desirable properties of large thermal conductivities and large breeding ratio [18-20].

\subsection{Nitrogen isotope separation process}

The separation of the nitrogen isotope has been studied since Urey et al. first succeeded in concentration of the nitrogen isotope of atomic weight 15 by using chemical exchange between ammonia gas and aqueous solutions of ammonium salts [21]. In their work, the 


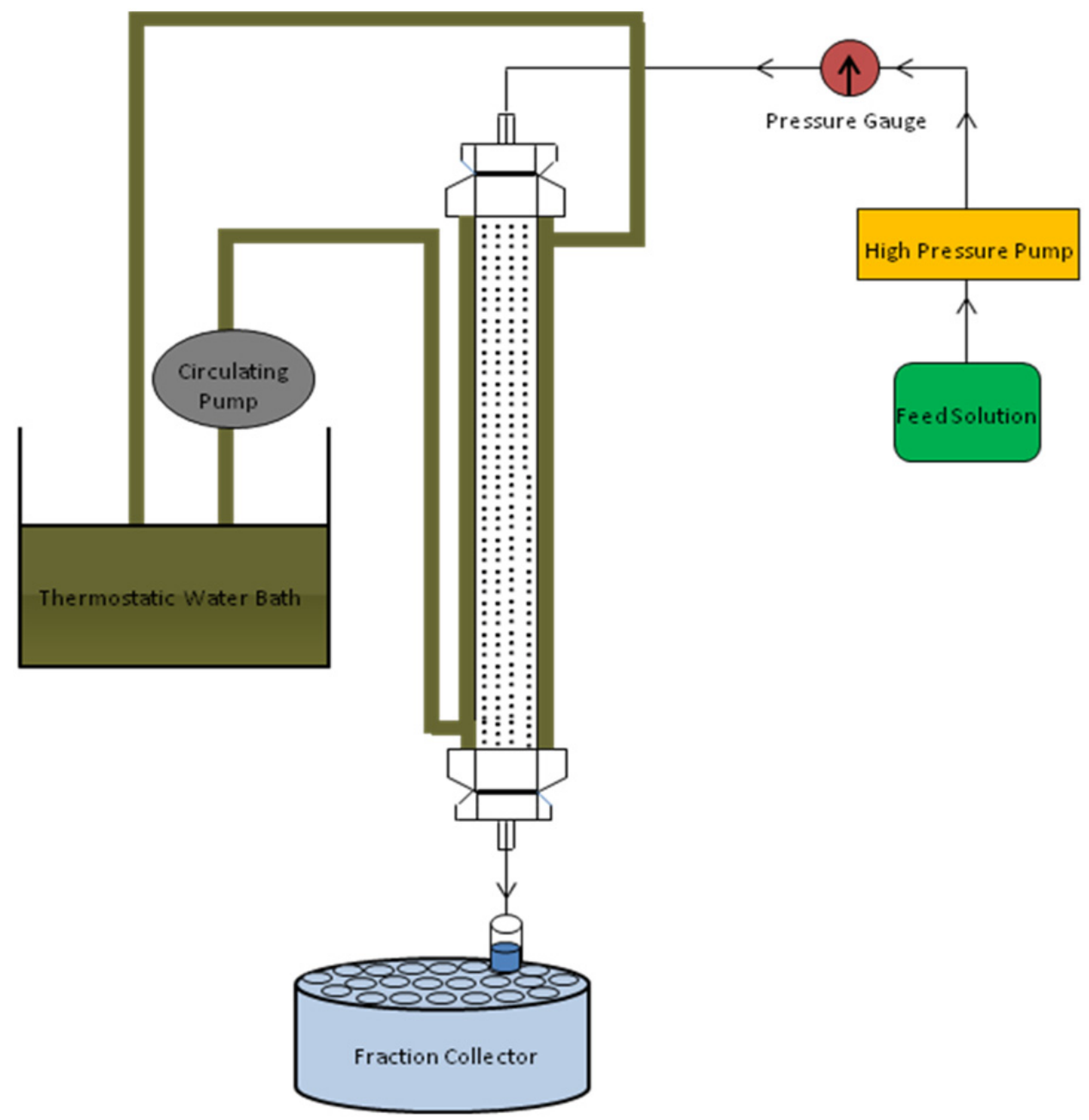

Figure 1. Schematic diagram of the apparatus for ion exchange separation

considerable quantities of material containing up to $75 \%$ atom of ${ }^{15} \mathrm{~N}$ was obtained by exchange between ammonia and aqueous ammonium nitrate solutions. Later, in 1955, Spinder and Taylor [22] developed the chemical exchange method involving the exchange between nitric acid and nitric oxide as Equation 1:

$$
{ }^{15} \mathrm{NO}+\mathrm{H}^{14} \mathrm{NO}_{3}={ }^{14} \mathrm{NO}+\mathrm{H}^{15} \mathrm{NO}_{3}
$$

The fractionation factor of the exchange reaction obtained at $1.0 \mathrm{M}$ nitric acid 1.062 is extremely favorable compared with the separation factors of most other possible separation methods of nitrogen isotopes by chemical system. In this process, the ${ }^{15} \mathrm{~N}$ concentrates in the nitric acid and thus ${ }^{15} \mathrm{~N}$ enriches in the lower part of the exchange column. In Japan, ${ }^{15} \mathrm{~N}$ has 
been commercially produced using this technique, which is called NITROX process. In Romania, Axente et al. have studied this process for thirty years using a laboratory-scale experimental plant [23]. Some other isotope separation methods such as distillation and thermal diffusion have also proved to be satisfactory for ${ }^{15} \mathrm{~N}$ separation [24-27].

Ion exchange process on nitrogen isotope separation has been studied since Spedding et al. first succeeded in the enrichment of ${ }^{15} \mathrm{~N}$ by cation exchange chromatography [6]. In their experiment, the separation factor of the isotopic exchange between the two phases of the dilute ammonium hydroxide solution and the cation exchange resin (Dowex 50-x12) in ammonium form was measured as $1.0257 \pm 0.0002$. A few years later, the studies on the nitrogen isotope separation by ion exchange were reported by two Japanese research groups Ishimori[28] and Kakihana [29]. Ishimori investigated the influence of operating temperature and concentration of ammonium solution on the isotope effects in a batch equilibrium system. Kakihana measured the separation factors of nitrogen isotopes for $\mathrm{NH}_{3}$ and $\mathrm{NH}_{4} \mathrm{Cl}$ in acetone water and ethanol water mixture systems. Afterwards, in 1980s, Park and Michaels studied the separation process developed by Spedding et al. under various operating conditions using columns packed with sulfonated polystyrene-divinylbenzene copolymers [30]. In the recent years, Krugrov et al. studied $\mathrm{NH}_{3}-\mathrm{NH}_{4}{ }^{+}$system using $\mathrm{SMB}$ (simulated moving bed) process for various flow rates under total reflux [31] and the theoretical analysis of separating nitrogen isotopes by ion exchange was reported by Aoki et al [32].

\subsection{Nitrogen isotopic analysis with mass spectrometry}

If an aqueous solution of ammonium hydroxide is placed in contact with a cation exchanger, the following exchange reaction occurs:

$$
{ }^{14} \mathrm{NH}_{4}{ }^{+}+{ }^{15} \mathrm{NH}_{4} \mathrm{OH}={ }^{15} \mathrm{NH}_{4}{ }^{+}+{ }^{14} \mathrm{NH}_{4} \mathrm{OH}
$$

the result of which, under equilibrium conditions, is that the heavy nitrogen isotope, by means of the ammonium ion, is distributed more abundantly in the solid phase, which is the cation exchanger. In order to multiply the elementary effect for separating ${ }^{15} \mathrm{~N}$, the following chromatographic separation process developed by Spedding was employed [6]: the ion exchanger bed was first converted to the hydrogen form by passing 2.0 M hydrochloric acid through the column until the bed was saturated. The bed was then rinsed with distilled water and an ammonium hydroxide solution of certain concentration was added. At the lower end of an ammonium band, the following reaction takes place:

$$
\mathrm{H}-\mathrm{R}+\mathrm{NH}_{4} \mathrm{OH} \rightarrow \mathrm{NH}_{4}-\mathrm{R}+\mathrm{H}_{2} \mathrm{O}
$$

Where $-\mathrm{R}$ denotes the fixed anion of cation exchanger. Since the equilibrium constant of this exchange reaction is on the order of $10^{9}$, the reaction is far to the right, resulting in an extremely sharp boundary. The ammonium ion comes to chemical equilibrium within the system very rapidly and isotopic equilibrium tends to be approached as the solution passes 
over the cation exchanger. In the frontal analysis, the effluent is collected in fractions at the bottom of the column when the ammonia solution of which the front part of the ammonium band is emerged. Prior to the collection, certain volumes of hydrochloric acid are initially added in the sampling tubes to prevent the ammonia from degassing. Since the ${ }^{15} \mathrm{~N}$ isotope is depleted in the front of the band, ${ }^{14} \mathrm{~N}$ isotope is enriched in the effluent.

In the displacement band chromatographic process, an ammonia solution is fed into the column until an ammonium adsorption band of the desired length is formed. The concentration of the fed ammonia solution was adjusted approximately at the concentration of the eluent in each operation. A caustic solution $(\mathrm{NaOH}$ or $\mathrm{LiOH})$ was then fed into the column to elute the ammonium band in the reverse breakthrough manner. At the top of the ammonium band, the following reaction takes place:

$$
\mathrm{NH}_{4}-\mathrm{R}+\mathrm{NaOH}(\mathrm{LiOH}) \rightarrow \mathrm{Na}(\mathrm{Li})-\mathrm{R}+\mathrm{NH}_{4} \mathrm{OH}
$$

which, having an equilibrium constant of about $10^{5}$, in turn guarantees a very sharp boundary at the rear end of the band. The ammonium ions are continuously released from the cation exchanger and converted to ammonium hydroxide by an equivalent of sodium (or lithium) ions which will be deposited at the rear edge of the band. The ammonium hydroxide solution moves down along the band until it reaches the front edge of the band. The diagram of the displacement band chromatographic process for separating nitrogen isotopes with the use of a cation exchanger is shown in Figure 2. The countercurrent movement of $\mathrm{NH}_{3}-\mathrm{NH}_{4}{ }^{+}$ions in two phases develops a longitudinal isotopic distribution profile within the band. The ${ }^{15} \mathrm{~N}$ isotope is enriched in the rear end of the band. The effluent of the rear part of the band was collected in fractions at the bottom of the column.

The volume of the fraction was determined by gravimetry, measuring the weight of the sample solution. The concentrations of ammonium and sodium (lithium) ions in the fractions were determined by an ion chromatography analyzer. The collected samples in the form of ammonium chloride were converted to nitrogen gas by adding a solution of potassium hypobromide $(\mathrm{KBrO})$. The reaction involved is as follows:

$$
2 \mathrm{NH}_{4} \mathrm{Cl}+3 \mathrm{KBrO}+2 \mathrm{NaOH}=\mathrm{N}_{2}+3 \mathrm{KBr}+2 \mathrm{NaCl}+5 \mathrm{H}_{2} \mathrm{O}
$$

The solution of potassium hypobromide was prepared by following process:

1. Add $36.5 \mathrm{~g}$ of sodium hydroxide and $2.2 \mathrm{~g}$ of potassium iodide into $170 \mathrm{~g}$ of distilled water.

2. Cool the solution 1 ) by ice and then $25 \mathrm{~g}$ of bromine is added with agitation.

3. The yellow colored solution obtained is left standing in a refrigerator for overnight.

The $\mathrm{KBrO}$ solution was stored in a refrigerator since potassium hypobromide is easily decomposed to potassium bromide and oxygen in light or by elevation of temperature. The Rittenberg was employed in order to prepare nitrogen gas samples for isotopic analysis and the apparatus of the nitrogen conversion system is shown in Figure 3. The preparation procedure of nitrogen gas samples is described below: 


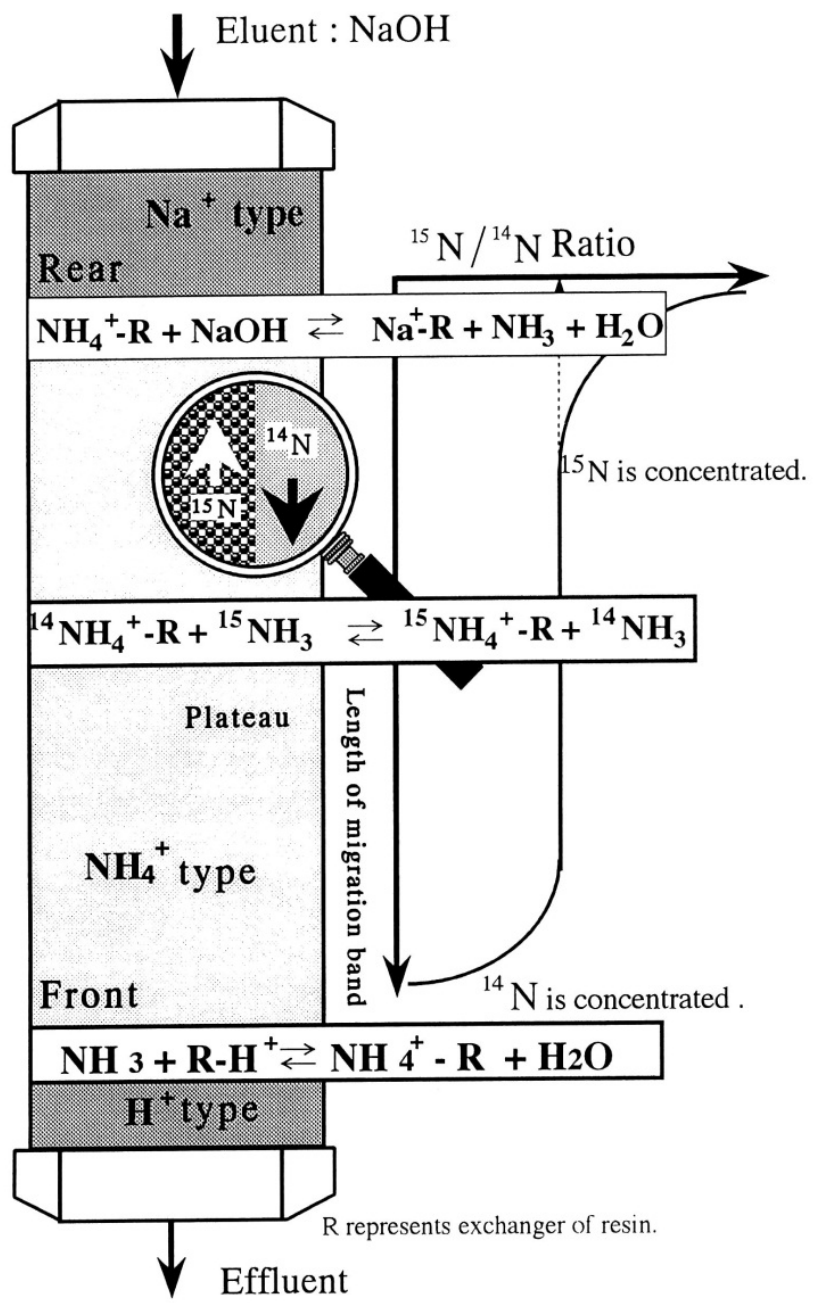

Figure 2. The diagram of the displacement band chromatographic process for nitrogen isotope separation

1. Put a certain volume of ammonium chloride sample $\left(0.1-1.0 \mathrm{~cm}^{3}\right)$ in one side and a stoichiometrically excess amount of potassium hypobromide in the other side of a Rittenberg glass tube A.

2. A cold trap D is cooled with liquid nitrogen to prevent contamination of water in the vacuum system during evacuation.

3. Freeze the solutions in the Rittenberg galss tube with liquid nitrogen.

4. Open the glass stopcocks $B$ and $C$ which are used to control the vacuum manipulations to pump out the air by an oil rotary pump. After the evacuation, the stopcock B is closed.

5. The liquid nitrogen vessel is then removed from the Rittenberg tube to admit the solutions to be melted. 
6. Freeze the solutions again and open the stopcock B so that the gas generated during the melting is evacuated.

7. After the evacuation, both stopcocks B and C are closed and Rittenberg tube is taken off from the conversion system.

8. Finally, nitrogen gas is obtained by mixing the solutions of ammonium chloride sample and potassium hypobromide in the Rittenberg tube.

9. The nitrogen gas sample is then introduced to the mass spectrometer for the isotopic analysis.

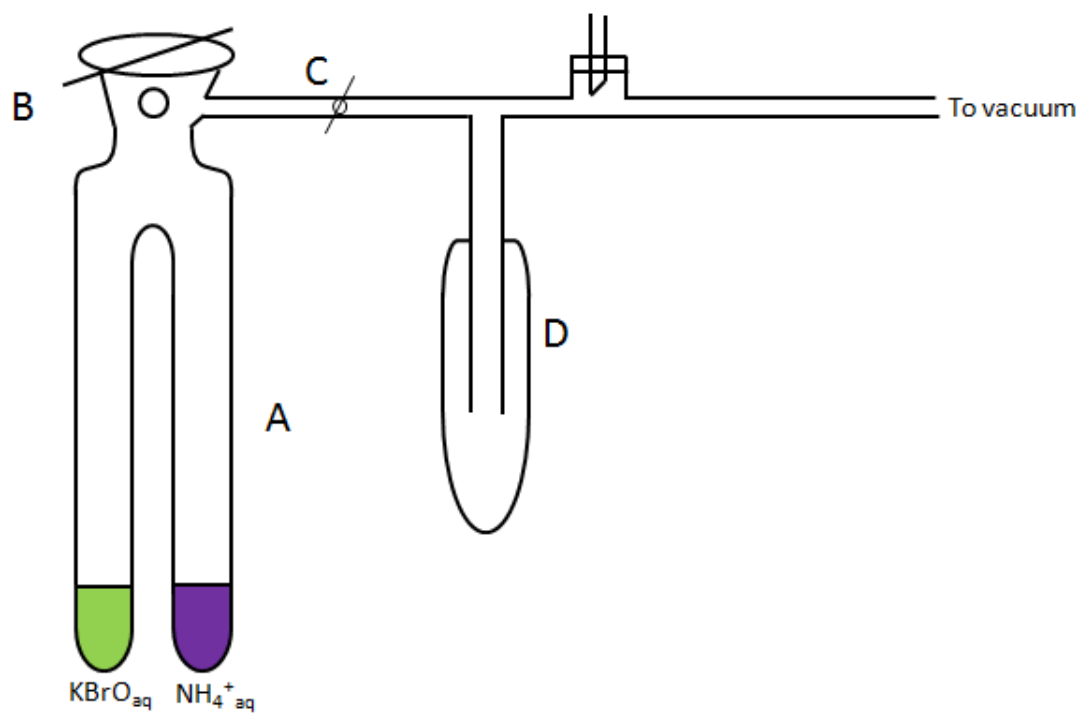

Figure 3. Schematic apparatus of the nitrogen gas conversion system

\section{The effect of migration distance to the nitrogen isotope separation}

The sulfuric strong acid cation exchange resin SQS-6(high porous type, cross linking 8\%, particle size 60-90 um in the form of $\mathrm{NH}_{4}^{+}$, provided by Asahi Chemical Industry Co., Japan) was packed uniformly in a pressurized glass column with a water jacket. The microscope view of $\mathrm{H}^{+}$type SQS-6 resin was shown in Figure 4. The ion exchange resin in the column preliminarily conditioned to the $\mathrm{H}^{+}$form with $2.0 \mathrm{M} \mathrm{HCl}$ solution, then pure water was feed into the top of the column to remove the free $\mathrm{H}^{+}$in the resins. $0.2 \mathrm{M} \mathrm{NH} \mathrm{H}_{4} \mathrm{OH}$ solution was feeded into the column to form $1.0 \mathrm{~m}$ ammonium adsorption band and the band was eluted by the displacing solution of $0.2 \mathrm{M} \mathrm{NaOH}$ in the reverse break-through manner. The flow rate and band velocity were controlled by feeding pump and the column pressure was monitored by high pressure pump. The temperature of the column was kept constant throughout the experiments by passing the thermostated water through the water jacket. The effluent, which emerged from the column, was collected using a fraction collector. In order to prevent the $\mathrm{NH}_{4} \mathrm{OH}$ samples from de-gassing, certain volumes of excess $\mathrm{HCl}$ solutions were added in the collection tubes prior to the samplings. Considering the long 
migration distance for the purpose of large scale production, it is necessary to regeneration column after the ammonium adsorption band pass through certain column. When the rear boundary of ammonium adsorption band enter into the next column, the first column was separated from the connection system and eluted by $2.0 \mathrm{M} \mathrm{HCl}$ for regeneration, the rinse volume was at least five times larger than that of the resin's volume. The concentrations of $\mathrm{NH}_{4}{ }^{+}$in the fractions were determined by ion chromatography analyzer and the mass peaks of ${ }^{14} \mathrm{~N}^{15} \mathrm{~N}$ and ${ }^{14} \mathrm{~N}^{14} \mathrm{~N}$ of the samples were measured with the mass spectrometer and the isotopic abundance of ${ }^{15} \mathrm{~N}$ was calculated from the ratio of the peak height.

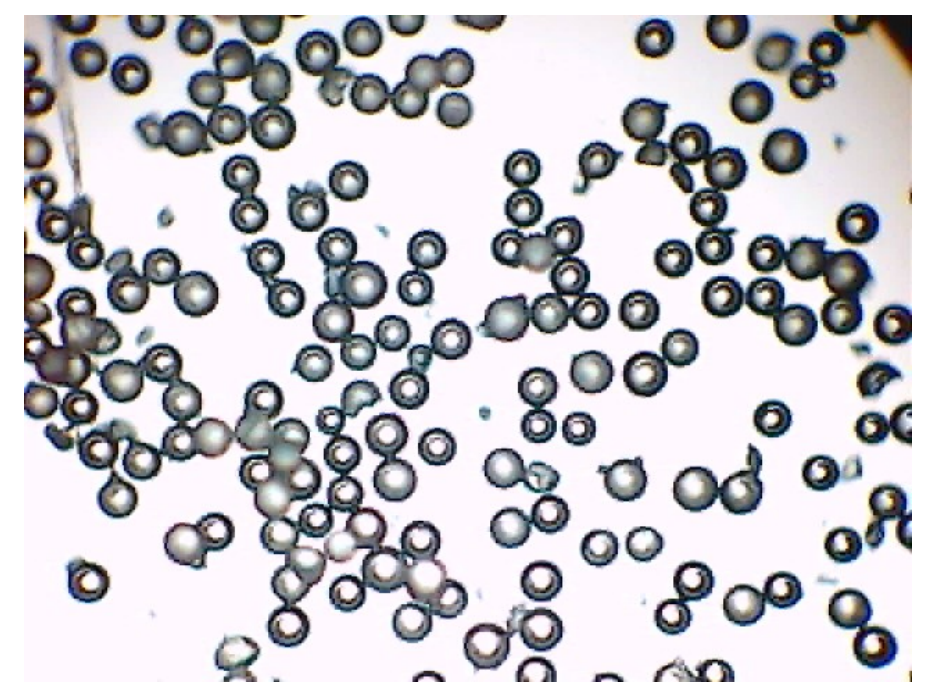

Figure 4. Microscope view of $\mathrm{H}^{+}$type SQS-6 resin

The separation of nitrogen isotopes by means of ion exchange resin is based on the isotopic fraction between ammonia in aqueous solution and ammonium ion in the ion-exchange resin as shown in the Equation 6. The single stage separation factor $\alpha$ or the separation coefficient $\varepsilon$ is defined by:

$$
\alpha=1+\varepsilon=\overline{[15 N]}[14 N] /[15 N] \overline{[14 N]}
$$

Where [] denotes the concentration of isotopes in the aqueous phase and $\overline{[]}$ the concentration of the isotopes in the resin phase.

In order to evaluate the chromatographic efficiency of nitrogen isotope enrichment, HETP (height equivalent to a theoretical plate) is introduced. HETP is usually calculated using the slope of the isotopic distribution curve in the steady-state of isotope separation after migration and is calculated by the following Equation 7:

$$
H=\frac{\varepsilon}{k}+\frac{1}{k^{2} L}
$$


Where $\mathrm{H}$ is the HETP and $\mathrm{L}$ is the migration length. The slope $\mathrm{k}$ is experimentally determined by using the following Equation 8:

$$
\operatorname{Ln}\left(r-r_{o}\right)=k(L-x)
$$

Where $r$ is the isotopic ratio of nitrogen sample and $r_{0}$ is the isotope ratio of feeding material. The term $(\mathrm{L}-\mathrm{x})$ is the distance between inner band location $\mathrm{x}$ and the rear or front boundary of which the migration length is L. Equation 8 indicates that plotting $\ln \left(\mathrm{r}-\mathrm{r}_{\mathrm{o}}\right)$ against (L-x) for the experimental data produces a linear line with the slope of $\mathrm{k}$.

Nitrogen isotope separations with different migration distance were performed and the experimental conditions were mentioned in Table 1.

\begin{tabular}{cccccc} 
Run & $\begin{array}{c}\text { Migration } \\
\text { distance }(\mathrm{m})\end{array}$ & $\begin{array}{c}\text { Column } \phi \\
(\mathrm{mm})\end{array}$ & $\begin{array}{c}\text { Eluent } \mathrm{NaOH} \\
\text { Conc. }(\mathrm{M})\end{array}$ & $\begin{array}{c}\text { Flow rate } \\
\left(\mathrm{cm}^{3} / \mathrm{min}\right)\end{array}$ & $\begin{array}{c}\text { Band velocity } \\
(\mathrm{m} / \mathrm{d})\end{array}$ \\
\hline 1 & 3 & 10 & 0.22 & 0.98 & 2.3 \\
2 & 4 & 10 & 0.21 & 0.99 & 2.3 \\
3 & 10 & 10 & 0.23 & 1.0 & 2.3 \\
4 & 30 & 10 & 0.25 & 0.98 & 2.3 \\
5 & 30 & 8 & 0.20 & 1.5 & 4.8
\end{tabular}

Table 1. Experimental conditions of chromatographic nitrogen isotope separation with different migration distance

The chromatographic elution curve for Run 4 as an example is given in Figure 5. It is seen that ideal displacement chromatograms are obtained and a sharp band boundary between the ammonium band and sodium band is maintained during the long migration at the current experimental conditions. The maintenance of the sharp band boundary is most important to obtain highly enriched isotope at the boundary region. Light isotope of ${ }^{14} \mathrm{~N}$ was enriched at the front boundary and the heavy isotope ${ }^{15} \mathrm{~N}$ was enriched at the rear boundary.

The two parameters of separation coefficient and HETP can be calculated from both front and rear boundary regions and the values were listed in Table 2.The observed $\varepsilon$ at front and rear boundary should be coincident each other. As shown in Table 2, the observed separation coefficients at front and rear boundary are in good agreement in each Run. The separation coefficient is an equilibrium factor and should be constant, being independent of the migration distance. It is confirmed from the results listed in Table 2 in the cases other than Run1. The small values of separation coefficient of Run1 are probably due to the feed material of $\left(\mathrm{NH}_{4}\right)_{2} \mathrm{SO}_{4}$ solution and one in $\mathrm{NH}_{4} \mathrm{SO}_{3}-\mathrm{R}$ in the ion exchange resin are resemble each other. In such case, isotope effects do not occur between the two chemical species. Due to this fact, the effective length of migration is reduced by approximately $20 \%$. This effect is reflected in the decrease in the separation coefficient in Run1.

It is also confirmed that the values of $\varepsilon$ obtained from Run 4 are slightly reduced. Probably this is due to the remixing in the middle of the adsorption band. Figure 5 showed the 
chromatographic profile of Run 4 . From the figure it can be seen that the isotopic plateau region is not seen, the enriched zone and the depleted zone are directly contacted because of the long migration. It should be noted that the values of $\varepsilon$ obtained from the experiments using natural nitrogen (Run 3) are practically the same as those observed by using enriched nitrogen (Run 5);

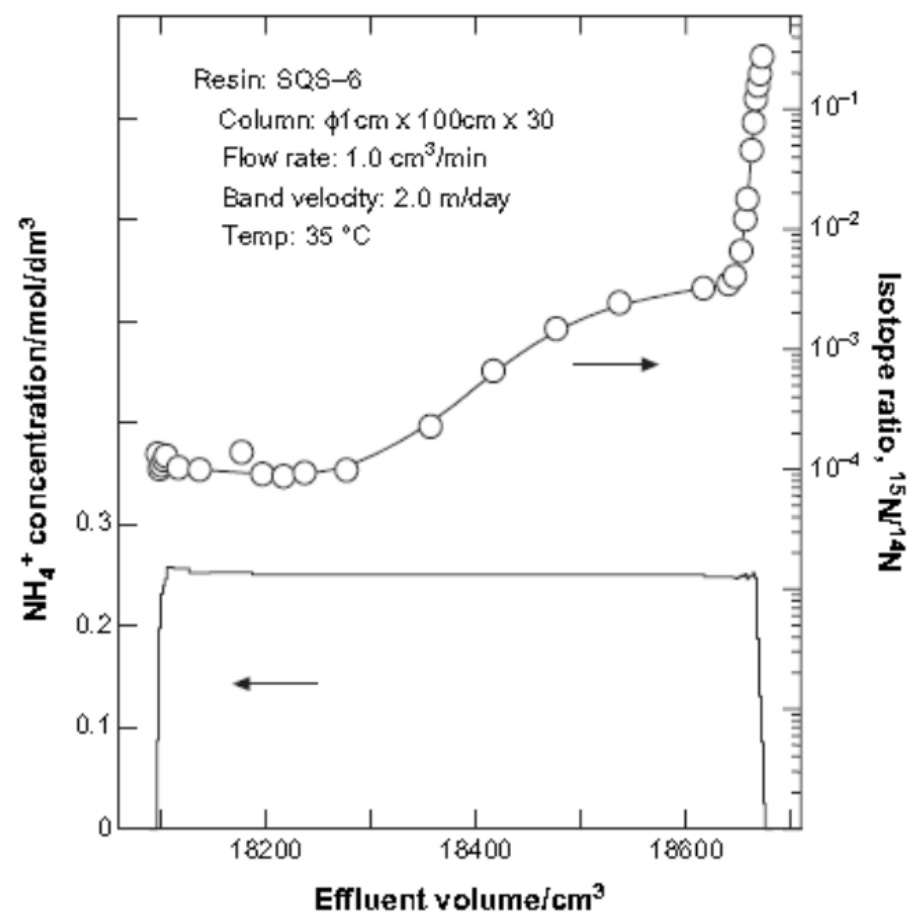

Figure 5. Chromatographic profiles of ammonium ion concentration and isotopic enrichment of $30 \mathrm{~m}$ migration distances.

\begin{tabular}{|c|c|c|c|c|c|c|c|}
\hline \multirow[t]{2}{*}{ Run } & \multicolumn{2}{|c|}{ Max. enrichment $(\%)$} & \multirow[t]{2}{*}{$\beta_{\text {rear }}=r_{\max } / r_{o}$} & \multicolumn{4}{|c|}{ Separation coefficient } \\
\hline & Front ${ }^{14} \mathrm{~N}$ & Rear ${ }^{15} \mathrm{~N}$ & & Front & Rear & Front & Rear \\
\hline 1 & 99.956 & 2.29 & 6.4 & 0.016 & 0.018 & 0.37 & 0.21 \\
\hline 2 & 99.987 & 3.91 & 11.1 & 0.025 & 0.025 & 0.74 & 0.19 \\
\hline 3 & 99.994 & 9.54 & 28.7 & 0.024 & 0.024 & 1.22 & 0.16 \\
\hline 4 & 99.990 & 21.55 & 78.9 & 0.021 & 0.019 & 2.6 & 0.16 \\
\hline $5^{a}$ & 99.143 & 99.678 & 77.4 & 0.023 & 0.022 & 0.39 & 1.58 \\
\hline
\end{tabular}

Run 5 used $80 \%{ }^{15} \mathrm{~N}$ as feed solution, of which $\mathrm{r}_{0}=4$.

Table 2. Experimental results of chromatographic nitrogen isotope separation with different migration distance 
The maximum enrichment $\beta$ values are defined as the local enrichment factor of ${ }^{15} \mathrm{~N}$ at the boundary as follows:

$$
\beta_{\text {rear }}=r_{\max } / r_{o}
$$

Where $r$ is the isotopic abundance ratio of ${ }^{15} \mathrm{~N}$ against ${ }^{14} \mathrm{~N}$. The values of $\beta$ calculated are listed in Table 2 and plotted as a function of migration distance in Figure 6. The slope of plots is unity, which means that the maximum enrichment $\beta$ values are proportional to the migration distance up to $30 \mathrm{~m}$. This fact suggests that the enrichment proceeds, forming an ideal shape of exponential enrichment curve at the band boundary region. In addition, it is quite interesting that $\beta$ value of Run 4 using natural nitrogen is in very good agreement with that of Run 5 where $80 \%$ enriched nitrogen was used as feed material. This information is important and useful for designing the enrichment plant based on the present method of ion exchange chromatography.

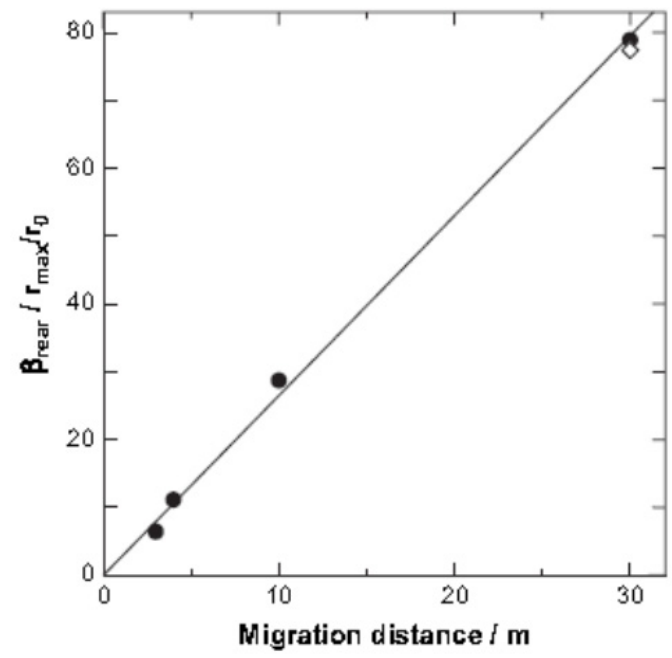

Figure 6. Correlation between migration distance and the maximum enrichment of ${ }^{15} \mathrm{~N}$

In order to evaluate the efficiency of nitrogen enrichment in chromatographic migration, HETP is introduced and the calculated values of HETP are listed in Table 2. HETP values are small enough in the rear band region of Runs $1-4$ where the enrichment of ${ }^{15} \mathrm{~N}$ is steadily proceeding. Similar phenomenon is observed in the front ${ }^{14} \mathrm{~N}$ enrichment zone of Run 5 . In general, the HETP values are small when the enrichment process does not reach the high level. On the other hand, saturation of isotope enrichment gradually takes place and slope of the enrichment curve sharply decreases when high enrichment is attained. This is the case for the enrichment of ${ }^{14} \mathrm{~N}$ in Runs 1-4 and the enrichment of ${ }^{15} \mathrm{~N}$ in Run 5. 
The results of Run 1-4 clearly indicate that the practical limit of ${ }^{14} \mathrm{~N}$ enrichment by the ion exchange packed column is $99.99 \%$. The reason for this limit has not yet been elucidated, but it is estimated that this limit of $99.99 \%$ may be applicable for the enrichment of ${ }^{15} \mathrm{~N}$ as well. So far, the target of ${ }^{15} \mathrm{~N}$ enrichment for nitride fuels is $99.9 \%$. The present work realized the enrichment of $99.678 \%{ }^{15} \mathrm{~N}$ in Run 5 . The results suggest that the $99.9 \%{ }^{15} \mathrm{~N}$ is attainable by ion exchange enrichment.

\section{The effect of cross linking to the nitrogen isotope separation}

Separation and concentration of a stable isotope from an isotopic mixture with natural occurrence is a very complex problem and usually, the isotopic separation coefficients of natural abundance are very low. In general, cation exchange process is a promising technique to produce highly enriched isotope due to the nature of small HETP value. Among the operating parameters in cation exchange process, cross linking is known as a decisive factor on the process of nitrogen isotope separation. It is interesting to evaluation the chromatographic performance of ${ }^{15} \mathrm{~N}$ isotope separation by ion exchange resin with different percentage of cross linking.

The cation exchange resins with different cross linking were synthesized from the law material of styrene and the synthetic method was given in Figure 7. When different cross<smiles>C=Cc1ccccc1C=C</smiles>

Styrene Divynylbenzene(DVB)

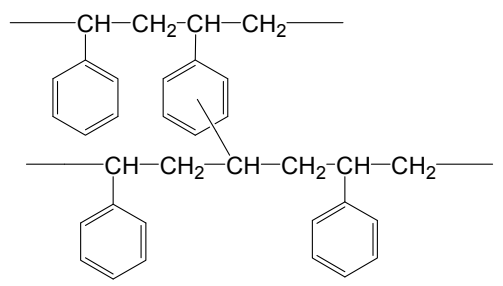

Styrene/DVB resin

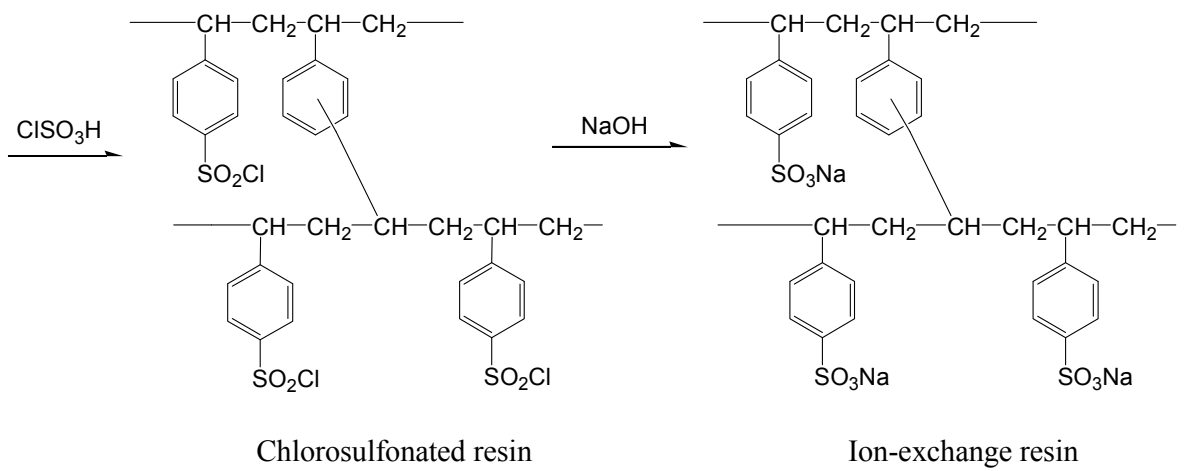

Figure 7. Synthetic method of the cation exchange resin with different cross linking percentage 
linking was synthesized, high cross linking means high divynybenzene (DVB) materials contained in the resin structure and this means the percentage of ion exchange functional group was decreased in the same amount resin. The relationship between ${ }^{15} \mathrm{~N}$ enrichment percentage and cross linking with $2.0 \mathrm{~m}$ migration was given in Figure 8. Enriched ${ }^{15} \mathrm{~N}$ isotopes were decreased from 0.93 to 0.68 when compared with the cross linking of twenty and forty percentage. Since high cross linking means low exchange capacity, it is reasonable that the ${ }^{15} \mathrm{~N}$ enrichment percentage is reduced with the increase of cross linking at the same given migration distances. SQS- 6 resin has $8 \%$ cross linking and ${ }^{15} \mathrm{~N}$ enrichment percentage was $1.56 \%$ when SQS-6 resin was performed under the same condition, and low cross linking resin has much higher enrichment ability than high cross linking resin.

The observed HETP values of different cross linking were plotted in Figure 9. It is seen in the figure that cross linking can affect the HETP value obviously. HETP value proportionally increase with the cross linking at the present work and low cross linking has much advantage for HETP. HETP value of $0.036 \mathrm{~cm}$ was obtained at the present system by using $20 \%$ cross linking and the present resin can be used for the large scale of nitrogen isotope industrial production.

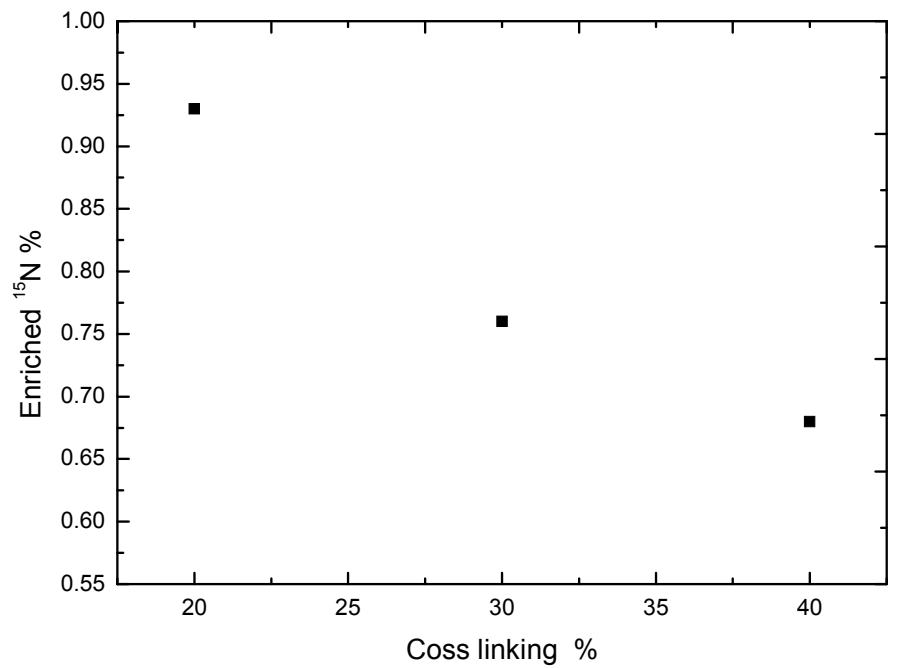

Figure 8. Relationship between enriched ${ }^{15} \mathrm{~N}$ and cross linking 


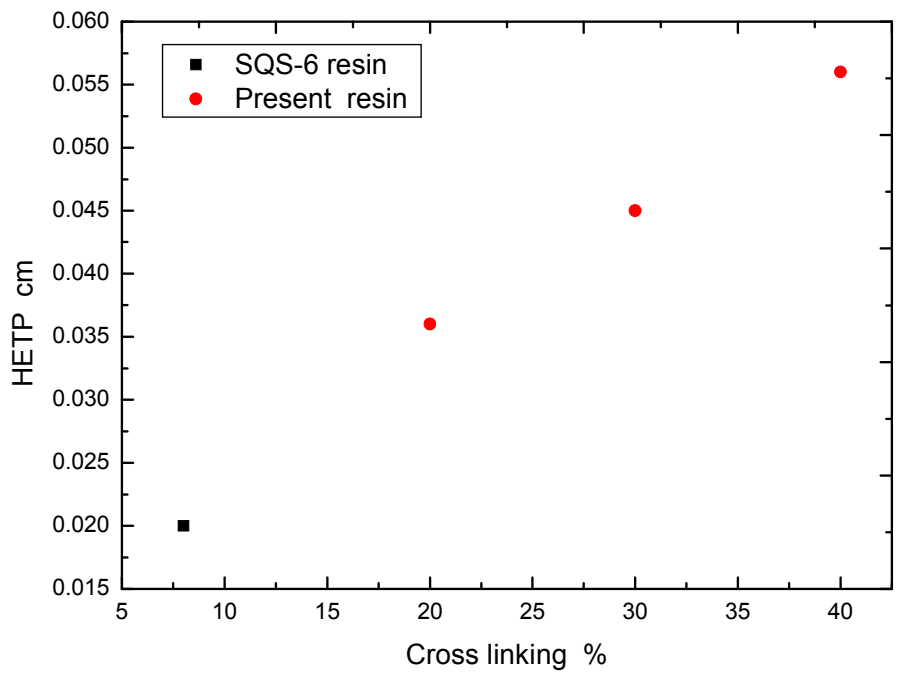

(Square dot was the result of SQS-6 resin)

Figure 9. Observed HETP values and cross linking

\section{Preparation of highly enriched ${ }^{15} \mathrm{~N}$ by chemical exchange}

The migration distance and cross linking can both affect the nitrogen isotope separation. It has relatively low separation coefficient by ion exchange chromatographic method when compared with that of NITROX method, it is very difficult to prepare $99.9 \%{ }^{15} \mathrm{~N}$ directly from natural abundance of $0.366 \%$. At the present stage of industrial factory, NITROX was used widely to produce various percentages of ${ }^{15} \mathrm{~N}$ products. It is very difficult to get highly enriched ${ }^{15} \mathrm{~N}$ by this method. In order to improve feasibility of this method, one good idea is to combine NITROX method and ion exchange chromatography. Starting from the natural abundance, NITROX has advantage to enrich ${ }^{15} \mathrm{~N}$ because of large separation coefficient; while when it reaches to relative highly enriched ${ }^{15} \mathrm{~N}$, due to the good merit of very small HETP value, the ion exchange chromatographic method is performed to get highly enriched ${ }^{15} \mathrm{~N}$.

In order to simulate the industry scale, large diameter column $(\phi=3.0 \mathrm{~cm})$ and high speed band velocity were used in this stage. $100 \mathrm{~g}$ commercial $\mathrm{NH}_{4} \mathrm{Cl}\left({ }^{15} \mathrm{~N}-80 \%\right)$ were feed into 3.0 $\mathrm{cm}$ diameter ion exchange columns. In order to shorten the ammonium adsorption time, the concentration of $\mathrm{NH}_{4} \mathrm{Cl}$ was adjusted to about $0.5 \mathrm{~mol} / \mathrm{dm}^{3}$ and the length of ammonium adsorption band was $138 \mathrm{~cm}$. Then $0.22 \mathrm{~mol} / \mathrm{dm}^{3} \mathrm{NaOH}$ was feed to the rear part of ammonium adsorption band to develop the adsorption band. The flow rate of feeding solution is $50 \mathrm{ml} / \mathrm{min}$ and the band velocity is $12 \mathrm{~m} / \mathrm{d}$. When the front boundary of 
ammonium adsorption band reached to twenty five meters, the three way valve was connected to the column for portion sampling. The sampling volume for each sample was about $0.5 \mathrm{ml}$ for every seven minute and it's corresponding to $5.0 \mathrm{~cm}$ of adsorption band for each sampling. These samples were analyzed by mass spectrometer for determine the nitrogen isotopic ratio as the result of the migration distance equal to 25 meters. When the rear boundary of ammonium adsorption band passes through the three way valve, the sampling was stopped. In order to keep the length of ammonium adsorption band constant and to avoid band diffusion, total amount of 3.0 L pure water with same flow rate was feed to the top of the column to develop the inside part of free ammonium molecular of adsorption band, the free ammonium molecular was move to the front boundary and readsorb again at the front boundary. The adsorption band moved forward $22.5 \mathrm{~cm}$ after $3.0 \mathrm{~L}$ pure water washing. Five days later, $\mathrm{NaOH}$ solution was feed again to develop the migration distance until fifty meters. The operation condition was the same with the first stage. When the front boundary of ammonium adsorption band was reached to fifty meters, three way valve was connected to the column for portion sampling. The sampling volume for each sample was about $0.5 \mathrm{ml}$ for every five minute and it's corresponding to $4.0 \mathrm{~cm}$ of adsorption band for each sampling. Before the rear boundary of ammonium adsorption band reach to three way valve, about $10 \mathrm{~cm}$ left the boundary, sampling was changed to whole fraction sampling and the volume of each sampling was $30 \mathrm{ml}$ and each corresponds to $1.0 \mathrm{~cm}$ of adsorption band, the sampling time was 45 second. Before sampling, excess amount of $\mathrm{HCl}$ was added to each bottle for neutralization. After the rear boundary pass through three way valve, 3.0 L pure water was feed to the top of column.

The large scale experimental conditions and results were listed in Table 3 and the chromatographic isotopic distribution curve of $25 \mathrm{~m}$ and $50 \mathrm{~m}$ migration distance were given in Figure 10 and Figure 11. During the long chromatographic operation, small amount ammonium ion exists in the tailing and may cause to the decrease the final isotope separation effect; this is the disadvantage of the long chromatographic operation. From the isotopic ratio curve of Figure 10 and Figure 11, the front boundary of ammonium adsorption band, the ${ }^{14} \mathrm{~N}$ isotopes could be continually enriched to over $99 \%$ which starting from $20 \%$. On the contrary, ${ }^{15} \mathrm{~N}$ isotope was enriched in the rear boundary regions steadily. Compare the results by Run1 and Run2 in Table 3, both are starting from $80 \%-{ }^{-15} \mathrm{~N} \mathrm{NH}_{4} \mathrm{Cl}$, the main differences are column diameter and flow rate. In Run3, high flow rate $(50 \mathrm{ml} / \mathrm{min})$ and 3.0 $\mathrm{cm}$ diameter column was used. The results show that at the rear band, both results have nearly the same values of separation coefficient $(0.022)$ and HETP $(0.16 \mathrm{~cm})$. At Run3, the highest percentage of enriched ${ }^{15} \mathrm{~N}$ was $99.756 \%$, higher than the value of $99.672 \%$ by Run2, even if the migration distance in Run3 is shorten than Run2. This means the speed of ion exchange between sodium and ammonium is very fast, and high flow rate does not affect to the HETP and separation coefficient in this operation systems. It is obviously that high flow rate has advantage to get highly enriched ${ }^{15} \mathrm{~N}$ and is much suitable to industrial operation. Run 3 and Run 4 were within the same experiment in a whole experiment; here it was divided into two parts. From the results of Run3 and Run4, ${ }^{15} \mathrm{~N}$ was steadily enriched with 


\begin{tabular}{|c|c|c|c|c|c|c|}
\hline Run No. & \multicolumn{2}{|c|}{ Run 1} & \multicolumn{2}{|c|}{ Run 2} & \multicolumn{2}{|c|}{ Run 3} \\
\hline Resin & \multicolumn{6}{|c|}{ SQS-6 high porous cation exchange resin } \\
\hline Feed solution & \multicolumn{2}{|c|}{$\begin{array}{c}0.19 \mathrm{M} \mathrm{NH}_{4} \mathrm{Cl}\left({ }^{15} \mathrm{~N}-\right. \\
80 \%)\end{array}$} & \multicolumn{2}{|c|}{$\begin{array}{c}0.50 \mathrm{M} \mathrm{NH} 4 \mathrm{Cl}\left({ }^{15} \mathrm{~N}-\right. \\
80 \%)\end{array}$} & \multicolumn{2}{|c|}{$\begin{array}{c}0.50 \mathrm{M} \mathrm{NH}_{4} \mathrm{Cl}\left({ }^{15} \mathrm{~N}-\right. \\
80 \%)\end{array}$} \\
\hline Effluent solution & \multicolumn{2}{|c|}{$0.20 \mathrm{M} \mathrm{NaOH}$} & \multicolumn{2}{|c|}{$0.23 \mathrm{M} \mathrm{NaOH}$} & \multicolumn{2}{|c|}{$0.24 \mathrm{M} \mathrm{NaOH}$} \\
\hline Temp. K & \multicolumn{6}{|c|}{308} \\
\hline Migration distance $\mathrm{m}$ & \multicolumn{2}{|c|}{30} & \multicolumn{2}{|c|}{25} & \multicolumn{2}{|c|}{50} \\
\hline Column diameter $\mathrm{mm}$ & \multicolumn{2}{|c|}{8} & \multicolumn{2}{|c|}{30} & \multicolumn{2}{|c|}{30} \\
\hline $\begin{array}{l}\text { Ammonium } \\
\text { adsorption band } \mathrm{m}\end{array}$ & \multicolumn{2}{|c|}{1.0} & \multicolumn{2}{|c|}{1.38} & \multicolumn{2}{|c|}{1.38} \\
\hline Flow rate $\mathrm{ml} / \mathrm{min}$ & \multicolumn{2}{|c|}{1.5} & \multicolumn{2}{|c|}{50} & \multicolumn{2}{|c|}{50} \\
\hline Band velocity $\mathrm{m} / \mathrm{d}$ & \multicolumn{2}{|c|}{4.8} & \multicolumn{2}{|c|}{11.1} & \multicolumn{2}{|c|}{14.4} \\
\hline $\begin{array}{l}\text { Adsorption capacity } \\
\mathrm{Q} \mathrm{mmol} / \mathrm{m}\end{array}$ & \multicolumn{2}{|c|}{74.3} & \multicolumn{2}{|c|}{1230} & \multicolumn{2}{|c|}{1250} \\
\hline Boundary & Front ${ }^{14} \mathrm{~N}$ & $\begin{array}{l}\text { Rear } \\
{ }^{15} \mathrm{~N}\end{array}$ & Front ${ }^{14} \mathrm{~N}$ & $\begin{array}{l}\text { Rear } \\
{ }^{15} \mathrm{~N}\end{array}$ & Front ${ }^{14} \mathrm{~N}$ & $\begin{array}{l}\text { Rear } \\
{ }^{15} \mathrm{~N}\end{array}$ \\
\hline Nitrogen $\%$ & 99.143 & 99.672 & 96.617 & 99.756 & 99.710 & 99.859 \\
\hline Separation coefficient & 0.023 & 0.022 & 0.022 & 0.022 & 0.023 & 0.023 \\
\hline Slope coefficient Ks & 0.6104 & 0.1541 & 0.5218 & 0.1569 & 0.4919 & 0.1599 \\
\hline HETP $\mathrm{cm}$ & 0.039 & 0.158 & 0.044 & 0.157 & 0.048 & 0.145 \\
\hline
\end{tabular}

Table 3. The experimental conditions and results for nitrogen isotope separation by using $80 \%{ }^{15} \mathrm{NH} \mathrm{H}_{4} \mathrm{Cl}$

the increase of the long migration distance, but the increasing trend becomes very low. For the first twenty five meter's migration, ${ }^{15} \mathrm{~N}$ has been enriched to $99.756 \%$, after another twenty five meter's operation, the final maximum enrichment percentage reached to $99.859 \%$. Enriched ${ }^{15} \mathrm{~N}$ is only increased $0.1 \%$ by another twenty five meter's migration, although the highly enriched regions became broaden and we can get much volume of ${ }^{15} \mathrm{~N}$ which the percentage is over $99.8 \%$. The separation coefficient of Run 3 and Run 4 is same because of the same operation condition; Run4 has relatively smaller HETP value than the value of Run 3. It indicates that long chromatographic operation has advantage and can steadily enrich ${ }^{15} \mathrm{~N}$ to very high percentage. The reason why the enriched ${ }^{15} \mathrm{~N}$ could not enrich to higher percentage in the present operation system may come from the stopping during the operation between Run3 and Run4. Because of the stopping by one week, the inner ammonium adsorption band may take remixing within the middle adsorption band. Highly enriched ${ }^{15} \mathrm{~N}$ at rear band will mix with middle level enriched ${ }^{15} \mathrm{~N}$ which was located in the middle band. If this operation restarts again, it will take long distance chromatographic operation to compensate the mixing and reach to the same percentage of ${ }^{15} \mathrm{~N}$ with twenty five meter's operation. From this result, the effective migration distance may be less than fifty meters. This can be confirmed by the analysis of the isotopic curve of Run 3 and Run 4 in Figure 10 and Figure 11. In Figure 10, there is a flat regions existing in the middle ammonium adsorption band, in this region, the ratio of ${ }^{15} \mathrm{~N} /{ }^{14} \mathrm{~N}$ is the same with 


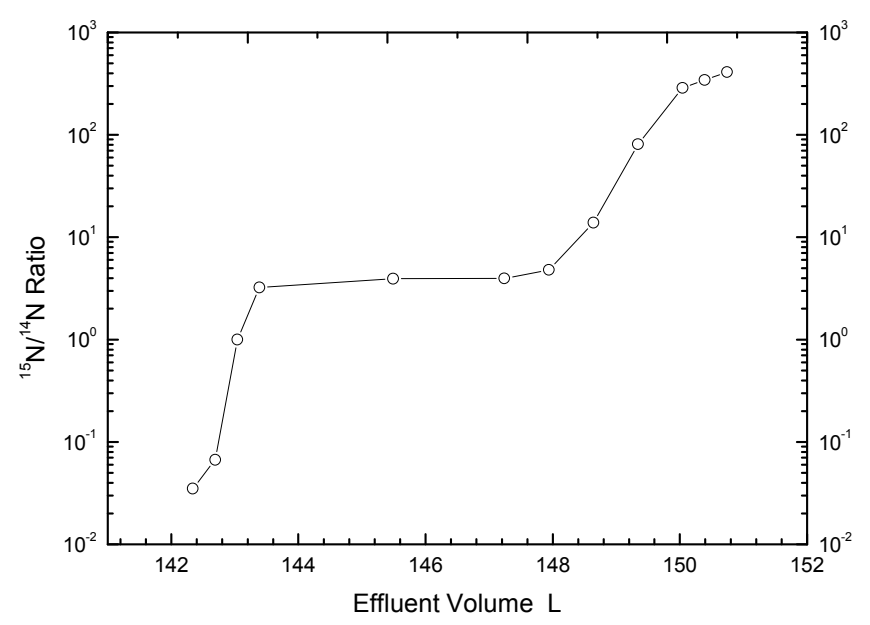

Figure 10. ${ }^{15} \mathrm{~N} /{ }^{14} \mathrm{~N}$ isotopic ratio (25 meter)

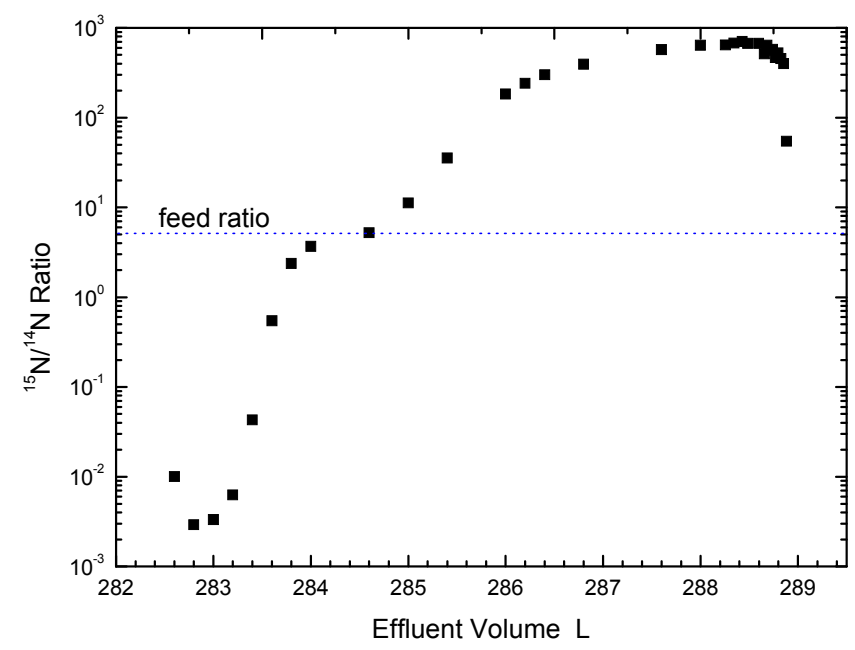

Figure 11. ${ }^{15} \mathrm{~N} / 14 \mathrm{~N}$ isotopic ratio (50 meter)

the original feeding solution, $80 \%$. At the rear boundary of the isotopic distribution curve, the slope of isotopic curve is still kept sharpness. In Figure 11, it was found that this flat region almost disappears and the isotopic ratio of ${ }^{15} \mathrm{~N} /{ }^{14} \mathrm{~N}$ was steadily increasing in the whole ammonium adsorption band. At the rear boundary region of the isotopic curve, there 
is a large amount of highly enriched ${ }^{15} \mathrm{~N}$ and the slope coefficient of isotopic distribution curve becomes flat. This is the evidence that by long chromatographic operation, highly enriched ${ }^{15} \mathrm{~N}$ was remixing again with the middle level enriched ${ }^{15} \mathrm{~N}$ in the middle position of ammonium adsorption band. The percentage and gram distribution of enriched ${ }^{15} \mathrm{~N}$ which along with ammonium adsorption band were listed in Table $4 .{ }^{15} \mathrm{~N}$ was mainly concentrated in the adsorption band which range from $64.5-152.03 \mathrm{~cm}$ in the adsorption band and 4.6 gram of highly enriched ${ }^{15} \mathrm{~N}(>99.82 \%)$ were successfully obtained.

\begin{tabular}{c|c|c}
\hline $\begin{array}{c}\text { Adsorption band length } \\
\mathrm{cm}\end{array}$ & $\begin{array}{c}\text { Percentage distribution of }{ }^{15} \mathrm{~N} \\
\%\end{array}$ & $\begin{array}{c}{ }^{15} \mathrm{~N} \\
\mathrm{~g}\end{array}$ \\
\hline $0 \sim 28.5$ & $<4.118$ & 0.0566 \\
\hline $28.5 \sim 33$ & $4.118 \sim 35.32$ & 0.3539 \\
\hline $33 \sim 46.5$ & $35.32 \sim 80.39$ & 2.2598 \\
\hline $46.5 \sim 64.5$ & $80.39 \sim 91.81$ & 3.2737 \\
\hline $64.5 \sim 82.5$ & $91.81 \sim 98.73$ & 3.5679 \\
\hline $82.5 \sim 123$ & $98.73 \sim 99.79$ & 8.1944 \\
\hline $123 \sim 147.3$ & $99.79 \sim 99.86$ & 4.6148 \\
\hline $147.3 \sim 152.03$ & $99.86 \sim 99.69$ & 0.8185 \\
\hline
\end{tabular}

Table 4. Percentage and gram distribution of enriched ${ }^{15} \mathrm{~N}$ along ammonium adsorption band by $50 \mathrm{~m}$ chromatography

\section{Conclusion of nitrogen isotope separation by ion exchange method and the comparison with NITROX separation method}

Long chromatographic operations were studied to nitrogen isotope separation by using $80 \%$ enriched ${ }^{15} \mathrm{~N}-\mathrm{NH}_{4} \mathrm{Cl}$. The main purpose was to get very highly enriched ${ }^{15} \mathrm{~N}$ isotope and to study how the flow rate and column diameter affect to nitrogen isotope separation. From the results obtained by each runs, it was confirmed that front and rear boundary could enrich ${ }^{14} \mathrm{~N}$ and ${ }^{15} \mathrm{~N}$ steadily to very high percentage. Since the feeding and elution solution are same for Run 1, Run 2 and Run 3, the same separation coefficients should be obtained no matter the migration distance. Due to the small amount of ammonium remained on the column, separation effect became slightly decrease with long chromatographic migration distance, especially by large diameter columns. When compared with the results of Run 2 and Run 3, the HETP value of Run 3 is smaller than Run2.

During the long operation, ${ }^{15} \mathrm{~N}$ can be steadily enriched to very high purity. Because the remixing phenomena take place, the highly enriched ${ }^{15} \mathrm{~N}$ will penetrated into the middle band, it decreased the maximum enrichment percentage of ${ }^{15} \mathrm{~N}$, but the highly enriched zones became broadened with long migration distance. The maximum percentage of enriched ${ }^{15} \mathrm{~N}$ was $99.859 \%$ and 4.6 gram of highly enriched ${ }^{15} \mathrm{~N}(99.82 \%)$ were successfully obtained by fifty meter's operation. 
Chemical exchange of nitrogen oxide system has been discussed for nitrogen isotope separation deeply in early times. Fifty years ago, W.Spindel and T. Taylor performed nitrogen isotope separation by chemical exchange (NITROX) with the condition of 15 $\mathrm{ml} / \mathrm{min}$ of $10 \mathrm{~mol} / \mathrm{dm}^{3}$ nitric acid, containing the natural ${ }^{15} \mathrm{~N}$ abundance [7]. They produced material containing $6.0 \mathrm{~g}$ of nitrogen analyzing $99 \%{ }^{15} \mathrm{~N}$ by twenty-five days continuously columns operation. Because of the large separation factor (1.055) by using NITROX method, most of industrial scale plants in the world are adopted with this method to produce nitrogen isotopes. As mentioned above, ion exchange method has the advantage of very small HETP, it can obtain very highly enriched nitrogen isotopes and no large amount of waste gas and liquid occurred, it can be widely used if combined with NITROX method when ${ }^{15} \mathrm{~N}$ has been enriched to the percentage of, for example, $80 \%$. One problem come from ion exchange method is the capacity of resin. The ion exchange $\mathrm{NH}_{4}-\mathrm{R} / \mathrm{NH}_{3}$ aq on cation exchange resin being inefficient for large scale production, according to the small flow rate accepted in the separation columns. That involves utilization of too large diameter separation columns with prohibitive amount of cation exchange resin.

Taking into account that the world market is about 20 to $40 \mathrm{Kg}{ }^{15} \mathrm{~N}$ annually, the supply of that isotope for nitride fuel production for nuclear power reactors (NPR) and accelerator driven system (ADS) would therefore demand an increase in production capacity by a factor of 1000 . For an industrial plant producing $100 \mathrm{t} / \mathrm{y}{ }^{15} \mathrm{~N}$, using present technology of isotopic exchange in NITROX system, the first separation stage of the cascade would be feed with $10 \mathrm{M} \mathrm{HNO}$ solution of $600 \mathrm{ml} / \mathrm{h}$ flow rate. If conversion of $\mathrm{HNO}_{3}$ into $\mathrm{NO}, \mathrm{NO}_{2}$, at the enriching end of the columns, would be done with gaseous $\mathrm{SO}_{2}$, for a production plant of $100 \mathrm{t} / \mathrm{y}{ }^{15} \mathrm{~N}$, a consumption of 4 million ton $\mathrm{SO}_{2} / \mathrm{y}$ and a production of $70 \% \mathrm{H}_{2} \mathrm{SO}_{4}$ waste solution of 4.5 million $\mathrm{ml} / \mathrm{y}$ are estimated. The reconversion of $\mathrm{H}_{2} \mathrm{SO}_{4}$ into $\mathrm{SO}_{2}$ in order to recycle of $\mathrm{SO}_{2}$ is a problem to be solved to compensate the cost of $\mathrm{SO}_{2}$, and to diminish the amount of $\mathrm{H}_{2} \mathrm{SO}_{4}$ waste solution. If it's considered that all ADS installations for minor actinides transmutation utilize nitride fuel with ${ }^{15} \mathrm{~N}$, the need of that isotope is about $4 \mathrm{t} / \mathrm{y}$, with a cost of 400 million USD (the price of ${ }^{15} \mathrm{~N}$ was considered 100 USD). It should be taken into consideration an important price reduction of ${ }^{15} \mathrm{~N}$ in order to make possible its utilization for large scale production of nitride fuel for NPRs and ADSs.

\section{Author details}

Xingcheng Ding* and Xunyue Liu Institute of Nuclear Agricultural Science, Zhejiang University, Hangzhou, P.R.China

\section{Acknowledgement}

The authors would like to express best thanks to Professor Yasuhiko Fujii, Dr. Tatsuya Suzuki and Dr. Masao Nomura, Research Laboratory for Nuclear Reactors, Tokyo Institute of Technology, Japan for their kindly guidance in technical and material supporting. 


\section{References}

[1] Ban Y, Nomura M, Fujii Y (2002)Chromatographic Separation of Lithium Isotopes With Silica Based Monobenzo-15-crown-5 Resin. J.nucl.sci.technol.39(3): 279-281.

[2] Ban Y, Nomura M, Fujii Y(2001)Isotope Effects of Strontium in Crown Ether Chromatography. Sep.sci.technol. 36(10):2165-2180.

[3] Ismail I, Fukami A, Nomura M, Fujii Y(2000)Anomaly of ${ }^{155} \mathrm{Gd}$ and ${ }^{157} \mathrm{Gd}$ Isotope Effects in Ligand Exchange Reactions Observed by Ion Exchange Chromatography. Anal. chem.72: 2841-2845.

[4] Matin M, Ismail I, Nomura M, Fujii Y(2002) Isotope Effects of Copper in Cu(II) Malate Ligand Exchange System Studied by Using Ion Exchange Displacement Chromatography, Sep.sci.technol. 37(9): 2129-2142.

[5] Nomura M, Higuchi N, Fujii Y(1996)Mass Dependence of Uranium Isotope Effects in the U(IV)-U(VI)Exchange Reaction. J.am.chem.soc. 118: 9127-9130.

[6] Spedding F, Powell J, Svec H(1955) A Laboratory Method for Separating Nitrogen Isotopes by Ion Exchange. J.am.chem.soc. 77(23): 6125-6132.

[7] Spindel W, Taylor T(1956)Preparation of $99.8 \%$ Nitrogen-15 by Chemical Exchange. J.chem.phys. 24: 626-627.

[8] Fujii Y, Aida M, Okamoto M, Oi T(1985)A Theoretical Study of Isotope Separation by Displacement Chromatography. Sep.sci.technol. 20(5\&6): 377- 392.

[9] Ishida $\mathrm{T}(2002)$ Isotope Effect and Isotope Separation: A Chemist's View. J.nucl.sci.technol.39(4): 407-412.

[10] Ishida T, Ono Y(2006)Early History of Chemical Exchange Isotope Enrichment and Lessons We Learn. J.nucl.sci.technol. 43(4): 391-399.

[11] Montanez A, Abreu C, Gill P, Hardarson G, Sicardi M(2009)Biological Nitrogen Fixation in Maize(Zea mays L.) by ${ }^{15} \mathrm{~N}$ Isotope-Dilution and Identification of Associated Culturable Diazotrophs. Biol fertil soils.45: 253-263.

[12] Sarr P, Khouma M, Sene M, Guisse A, Badlane A, Yamakawa T(2008)Effect of Pearl Millet-Cowpea Cropping Systems on Nitrogen Recovery, Nitrogen Use Efficiency and Biological Fixation Using the ${ }^{15} \mathrm{~N}$ Tracer Technique. Soil. sci .plant. nutr. 54: 142-147.

[13] Bosshard C, Sorensen P, Frossard E, Dubois D, Mader P, Nanzer S, Oberson A(2009)Nitrogen Use Efficiency of ${ }^{15} \mathrm{~N}$-labelled Sheep Manure and Mineral Fertilizer Applied to Microplots in Llong-term Organic and Conventional Cropping Systems. Nutr. cycl.agroecosyst.83:271-287.

[14] Hood R(2001)Evaluation of A New Approach to the Nitrogen-15 Isotope Dilution Technique, To Estimate Crop N Uptake From Organic Residues in The Field. Biol.fert.soils. 34(3): 156-161.

[15] Sorensen P, Thomsen I(2006)Separation of Pig Slurry and Plant Utilization and Loss of Nitrogen-15-labeled Slurry Nitrogen. Soil. sci. soc. am. j. 69: 1644-1651. 
[16] Thomsen I, Kjellerup V, Jensen B(1997)Crop Uptake and Leaching of ${ }^{15} \mathrm{~N}$ Applied in Ruminant Slurry With Selectively Labeled Faeces And Urine Fractions. Plant soil.197: 233-239.

[17] Gutser R, Ebertseder Th, Weber A, Schraml M, Schmidhalter U(2005)Short-term and Residual Availability of Nitrogen After Long-term Application of Organic Fertilizers On Arable Land. J. plant. nutr. soil sci. 168(4):439-446.

[18] Ding X, Suzuki T, Nomura M, Aida M, Fujii Y(2005)Nitrogen Isotope Enrichment For Nitride Fuel by Using Hybrid Chemical Exchange Process. Prog.nucl.Energy. 47: 420425.

[19] Ding X, Kaneshiki T, Arima M, Nomura M, Suzuki T, Fujii Y(2008) High Enrichment of ${ }^{15} \mathrm{~N}$ Isotope by Ion Exchange For Nitride Fuel Development. Prog. nucl. Energy. 50: 504509.

[20] Ding X, Nomura M, Suzuki T, Fujii $\mathrm{Y}(2008)$ High Enrichment of ${ }^{15} \mathrm{~N}$ by Chromatographic Chemical Process.J.Chroms.A 1201: 65-68.

[21] Urey H, Huffman J, Thode H, Fox M(1937)Concentration of ${ }^{15} \mathrm{~N}$ by Chemical Methods. J. chem. phys. 5: 856-867.

[22] Spindel W, Taylor T(1956) Preparation of $99.8 \%$ Nitrogen-15 by Chemical Exchange. J. chem. phys. 24: 626-627.

[23] Axente D, Baldea A, Abrudean M(1992) Isotope Separation by Chemical Exchange, Proceedings of the International Symposium on Isotope Separation and Chemical Exchange Uranium Enrichment. In Fujii Y, Ishida T, Takeuchi K, editors. Bulletin of the Research Laboratory for Nuclear Reactors, Tokyo Institute of Technology. pp 357-367.

[24] Mills T, Garcia M, Vandervoort R, McInteer B(1989) A Chemical Exchange System for Isotopic Feed to a Nitrogen and Oxygen Isotope Separation Plant. Sep. sci.technol.24:415-428.

[25] Kendall J(1942)Separation of Isotopes and Thermal Diffusion. Nature .150:136-140.

[26] Majumdar S(1951)The Theory of The Separation of Isotopes by Thermal Diffusion. Phys. review, 81(5): 844-848.

[27] Spindel W, Taylor T (1955) Concentration of Nitrogen-15 by Chemical Exchange in a Thermal Diffusion Column.J. chem. phys. 23(7): 1318-1322.

[28] Tatsukiro I(1960)The Nitrogen Isotopic Equilibrium Between Ammonia and Ammonium Ion. Bull.chem.soc.jpn. 33: 516-519.

[29] Kakihana H(1963)A Fundamental Study On the Ion Exchange Separation of Lithium, Nitrogen and Uranium Isotopes. J. DE chimie physique ET DE physico-chimie biologique 60(1-2): 81-88.

[30] Park W, Michaels E(1988)Separation of Nitrogen Isotopes by Displacement Band Chromatography. Sep. sci. technol. 23: 1875-1889.

[31] Kruglov A, Andreev B, Pojidaev Y(1996)Continuous Isotope Separation in Systems With Solid Phase. II. Separation of Nitrogen Isotopes With Use of Ion-exchange Resin. Sep. sci. technol.31: 471-490. 
[32] Aoki E, Kai T, Fujii Y(1997)Theoretical Analysis of Separating Nitrogen Isotopes by Ionexchange . J. nucl. sci. technol. 34(3): 277-282. 